\title{
Leseverstehen im Fremdsprachenunterricht mit Hilfe der pragmalinguistischen Typologie
}

\author{
Erzsébet Szabó - Eva Stranovská - Ol'ga Wrede
}

\begin{abstract}
Reading comprehension in foreign language teaching with the help of the pragmalinguisitc typology

Abstract

This paper deals with the analysis of foreign language texts with the help of the pragmalinguistic typology which plays an essential role in describing the cognitive level. It also describes how it is possible to improve the reading skills of the students based on the pragmalinguistic typology which has a decisive influence in developing the pupil's communicative competence. Pragmalinguistics is intended to enable an individual to recognize different texts and to understand exactly what the author wants to highlight. For this it is necessary to know how to produce semantically and syntactically acceptable sentences with the appropriate choice of words. Our basis is the cognitive theory of Eve Sweetser. According to her teaching an utterance is based on three cognitive linkage levels: the propositional, the epistemic and the speech act level.
\end{abstract}

Keywords: reading comprehension; pragmalinguistic typology; cognitive linkage level

Schlüsselwörter: Leseverstehen; pragmalinguistische Typologie; kognitive Verknüpfungsebene

Subject-Affiliation in New CEEOL: Language and Literature - Theoretical Linguistics - Pragmatics

DOI: $10.36007 /$ eruedu.2020.3.048-059

\section{Einleitung}

Für diejenigen, die eine Fremdsprache lernen, ist das Lesen eine geeignete und entscheidende Strategie, die Zielsprache und somit auch die Zielkultur kennenzulernen. Durch das Lesen können sie sich in die Struktur einer Fremdsprache vertiefen und begreifen wie die Zielsprache funktioniert. Das Lesen hilft ihnen auch, ihren Wortschatz zu erweitern, ihre Grammatikkenntnisse auszubauen, ihre Ausdrucksfähigkeiten zu verbessern und fließend zu lesen und zu schreiben.

Das Lesen in der Fremdsprache ist eng mit dem Lesen in der Muttersprache verbunden. Der Leser verarbeitet die erhaltenen Informationen in seiner Muttersprache und das Leseverstehen in der Fremdsprache wird von der Muttersprache 
beeinflusst. Darüber hinaus kann man von den Studierenden, die Schwierigkeiten beim Lesen in ihrer Muttersprache haben, auch keine guten Leistungen beim Lesen in der Fremdsprache erwarten.

Heutzutage lesen ganz viele Menschen nur selten, sowohl in ihrer Muttersprache als auch in der Fremdsprache, weil sie in geringem Maße die Bedeutung und den Sinn der Texte verstehen. Im Lesen wird kulturelles Verständnis möglich, weil der Text die kulturelle Basis des Zielsprachenlandes repräsentiert. Aus diesem Grund wird in diesem Beitrag das Leseverstehen mit der Interkulturalität verbunden.

Der vorliegende Artikel beschäftigt sich vor allem mit der Bedeutung der Lesefertigkeit in der Fremdsprache und auch mit dem Textverstehen im Fremdsprachenunterricht. Daneben wird in dieser Forschungsarbeit darauf hingewiesen, wie die Lesefertigkeit bei den Studenten unter Zuhilfenahme der Pragmalinguistik entwickelt werden kann, die eine sehr wichtige Rolle in der Herausbildung der kommunikativen Kompetenz des Individuums spielt. Die Pragmalinguistik dient als ein Mittel, mit dessen Hilfe man unterschiedliche Texte erkennen und aus den Texten genau das verstehen kann, was der Autor hervorheben und betonen möchte.

\section{Bedeutung der Lesefertigkeit in der Fremdsprache}

Die Lesefertigkeit wird als eine der rezeptiven Kommunikationsfertigkeiten angesehen, bei der die empfangenen Informationen im geschriebenen Text enthalten sind und der Empfänger davon beim Lesen erfährt (Hirschnerová, 2012, 2). Dieser Begriff wird vor allem beim Lesen in der Fremdsprache verwendet, bei der die Wahrnehmung des grafischen Bildes des sprachlichen Ausdrucks, die Bestimmung von Buchstaben, Wörtern und Sätzen sowie das Gesamtverstehen des Textes sehr wichtig sind (Terpitková 2013, 8). Der Begriff Lesefertigkeit wird sehr häufig mit der Lesekompetenz verwechselt und sie werden oft als Synonyme verwendet.

Die Lesekompetenz bezeichnet die komplexe Entwicklung der Lesefertigkeit, die für eine effektive und ausführliche Textarbeit unerlässlich ist. Die Lesekompetenz wird häufig im Zusammenhang mit dem Lesen in der Muttersprache erwähnt. Die Lesefähigkeit ist nach Kovárová und Kurtulíková $(2010,12)$ eine elementare Eigenschaft des Individuums, eine Voraussetzung für seine vielseitige Entwicklung und für seine gesellschaftliche Durchsetzungsfähigkeit. Im Leseprozess geht es um eine wechselseitige Beziehung zwischen dem Text und dem Leser. Während des Lesens verwendet der Leser angemessene Methoden zur Identifizierung von Zeichen, versucht, die Bedeutung des Textes zu finden und schließlich kann er neue Informationen mit seinen vorhandenen Kenntnissen kombinieren.

Das Lesen hat mehrere positive Seiten. In erster Linie bereichert es den aktiven Wortschatz des Individuums, entwickelt seine Sprachfähigkeit und beeinflusst das emotionale Erleben, die Wahrnehmung und die ästhetische Orientierung des Lesers. Daneben fördert es das abstrakte Denken und hilft beim Hören und der visuellen Fixierung grammatischer Phänomene (Clarke-Truelove-Hulme-Snowling, 2014, 1-2; Gadušová, 2004, 77-78; Kovárová-Kurtulíková, 2010, 12). 
Bei der Entwicklung des Leseverstehens in einer Fremdsprache ist es wichtig, die funktionalen und die komplexen Aspekte der Lesekompetenz nach Gavora (2003, 8-9) zu berücksichtigen, die nicht nur in der Muttersprache, sondern auch in der Fremdsprache angewendet werden können. Bei dem funktionalen Aspekt liegt der Schwerpunkt auf der Fähigkeit des Individuums, verschiedene Informationen aus dem gelesenen Text zu verarbeiten und die erworbenen Kenntnisse in anderen Situationen zu verwenden. Der komplexe Aspekt der Lesekompetenz bedeutet die Fähigkeit, kreativ zu lesen und zu schreiben. Außerdem ermöglicht er den Studierenden, Texte verschiedener Genres und Formen zu interpretieren und kritisch zu bewerten. Der komplexe Aspekt erfordert nicht nur den Einsatz der rezeptiven (Hören, Lesen), sondern auch der produktiven (Sprechen, Schreiben) Kommunikationsfertigkeiten, die unerlässlich beim Erlernen einer Fremdsprache sind und mit deren Hilfe die Studierenden sich besser im Textverstehen vertiefen können (Gavora, 2008, 51; Liptáková-Cibáková, 2013, 11-12).

\subsection{Das Textverstehen und sein Prozess}

Das Verstehen ist eine genetisch gegebene menschliche Tätigkeit. Das Textverstehen ist eine inhaltliche und ästhetische Interpretation des Textes. Es bedeutet die Suche nach der Bedeutung des Textes (Gavora, 2008, 51).

Das Textverstehen ist eine entscheidende Voraussetzung für den „Einstieg“ des Studenten in den Lehrstoff. Das Lernen ist nicht effektiv, sinnvoll und vertrauenswürdig, wenn das Verstehen fehlt. Aus den Erfahrungen der Lehrenden geht hervor, dass die Studierenden den Lernstoff ganz oft nur einpauken, ohne ihn zu verstehen, um ihn dann nur mehr oder weniger mechanisch zu reproduzieren. Den auf diese Weise gelernten Lernstoff versteht der Einzelne nicht und weiß nicht, wie er die Theorie in den konkreten Situationen anwenden soll.

Das Leseverstehen betrachtet man als Grundlage für die Wahrnehmung der Welt und es dient auch als ein wichtiges Hilfsmittel bei der Aneignung des Lernstoffes. Es ist eine Fertigkeit, die das Individuum bei der Entwicklung der Lesekompetenz erwirbt. Ohne Verstehen ist das Individuum unschöpferisch, nicht selbstständig, lebt in Unwissenheit, denkt nicht und ist leichter zu manipulieren. Das Verstehen von schulischen und außerschulischen Texten spielt eine sehr wichtige Rolle bei der psychischen Entwicklung der Studenten. Die Texte bieten innen neue Informationen, zwingen sie zum Nachdenken, helfen den Individuen Beziehungen auszubilden und Meinungen über die Welt zu bilden (Koncepcia rozvoja kultúrnych a čitatel'ských kompetencii detí prostredníctvom kultúrnej výchovy s ciel'om zriadenia Centra literatúry pre deti a mládež a podpory číania, 2017).

Mit dem Leseprozess selbst haben sich schon mehrere Linguisten beschäftigt, wie zum Beispiel Ehlers (2006, 1992), Westhoff (1991), Bechtel (1984) und Solmecke (1997). Ehlers (2006) hat in ihrer Forschung (Entwicklung von Lesekompetenz in der Fremdsprache) einige Teilfertigkeiten dargestellt, die eine wichtige Rolle im Prozess des Leseverstehens spielen.

Nach Ehlers (2006, 31-37) muss der Leser die Informationen aus dem gelesenen Text mit seinen Erfahrungen verbinden. Unter dem Begriff Erfahrung versteht 
man Kenntnisse über die Welt, Normen, Vorschriften, gesellschaftliche Konventionen, Traditionen und Geschichte. Die Wörter im Text aktivieren die Vorkenntnisse des Lesers, deshalb ist die Wortschatzarbeit sehr wichtig, um die Lesefertigkeit zu verbessern. Im engeren Sinne basiert das Textverstehen aber nicht nur auf den Kenntnissen des Individuums, sondern auf der Inferenz. Der Leser sollte in der Lage sein, zwischen den Zeilen zu lesen und überlegen, was die Darsteller in der Geschichte denken und darüber nachdenken, was vor den Ereignissen hätte passieren können oder wie die Geschichte weitergehen könnte. Daneben sollte der Leser auch in einem gewissen Maße über Gedankenflexibilität verfügen, die die Entwicklung der metakognitiven Strategien unterstützt und eine Vertiefung in den Text ohne Frustration von unbekannten Vokabeln und Sprachstrukturen ermöglicht (Stranovská, 2011, 55).

Beim Textverstehen ist es auch unentbehrlich, die Grammatik und den Wortschatz des Textes zu verstehen. Zu schwache Beherrschung des Wortschatzes und mangelnde grammatische Kenntnisse schränken die Entwicklung der Lesekompetenz ein. Typisch für dieses Lesen ist das kürzere Leseintervall, das heißt, Fähigkeit, Informationen aus dem Text im Kurzzeitgedächtnis zu bewahren (Ehlers, 2006, 31-37).

Die oben beschriebenen Teilfertigkeiten bilden die Grundlage, die möglichen Probleme beim Textverstehen zu identifizieren, wertvolle Ratschläge sowohl den Lehrenden als auch den Studierenden zu geben und das Leseverstehen in der Fremdsprache zu verbessern.

\section{Pragmalinguistik im Kontext des Textverstehens}

Im Rahmen des Fremdsprachenunterrichts spielt die Pragmalinguistik eine sehr wichtige Rolle, weil sich die Kommunikation in textuellen Kontexten realisiert. Beim Textverstehen ist es unerlässlich zu wissen, wie man syntaktisch und semantisch korrekte Sätze produziert. Daneben muss man auch darauf achten, welche sprachlichen und außersprachlichen Mittel man gebraucht, damit der Gesprächspartner versteht, was man ausdrücken möchte (Günther-Günther, 2007, 76; HufeisenNeuner, 1999, 43).

Die Pragmalinguistik im Kontext des Textverstehens kann mit Hilfe der pragmalinguistischen Typologie beschrieben werden. Die pragmalinguistische Typologie ist eng mit der kognitiven Theorie von Sweetser (1990) verbunden, die die kognitive Ebene analysiert. Ihre Lehre geht von der Theorie des Linguisten George Lakoff (1980) und des Philosophen Mark Johnson (1980) aus, und für diese Theorie ist charakteristisch, dass die einzelnen lexikalischen Einheiten nicht nur eine konkrete Bedeutung haben, sondern mit Hilfe eines kognitiv-metaphorischen Prozesses auch ihr abstrakter Gebrauch beschrieben werden kann (Ferraresi, 2011, 129-130). 
Laut Sweetser's kognitiver Theorie lässt sich feststellen, dass eine Äußerung auf drei kognitiven Lesarten/Verknüpfungsebenen erklärt werden kann:

- die propositionale Ebene

- die epistemische Ebene

- die sprechaktbezogene Ebene (Gagel, 2015, 56).

Die erste Verknüpfungsebene verkörpert die propositionale Ebene. Die propositionale Ebene ist präsent, wenn die Aussage einen so genannten konstativen Charakter hat, wie zum Beispiel Berichte, Beschreibungen oder Schilderungen. Typische Merkmale der propositionalen Ebene sind adverbiale Bestimmungen der Zeit, Indikativformen und vergangenheitsbezogene Tempusformen (Gagel, 2015, 29; Weiss, 2016, 22).

Man spricht über die epistemische Verknüpfungsebene, wenn das externe Konnekt ein Evaluativum, ein Estimativum oder ein Identifikativum enthält. Das Evaluativum beschreibt solche Aussagen, die vor allem Bewertungen und Werturteile ausdrücken. Charakteristische Merkmale der Evaluativa sind evaluative Verben, Adjektive und Substantive.

Das Estimativum kann durch Behauptungen, Vermutungen oder Annahmen zum Ausdruck gebracht werden. Zu den Indikatoren der Estimativa gehören Adverbien wie „wahrscheinlich", „möglicherweise“, „vielleicht", „offenbar", „allerdings" oder „nämlich“.

Der letzte Subtyp ist das Identifikativum, das vor allem Gefühle und Hoffnungen des Sprechers ausdrückt. Zu den typischen Indikatoren des Identifikativums zählt man die Adverbien wie „glücklicherweise“, „hoffentlich" oder „leider" (Gagel, 2015, 29).

Die pragmatische Funktionalität lässt sich auch mit verschiedenen Modalitätsmarkern bestimmen, die in erster Linie auf die epistemische Verknüpfungsebene hinweisen. Zu den Modalitätsmarkern gehören die Modalverben, die objektive oder subjektive Modalität von der Seite des Sprechers ausdrücken. Zu den weiteren Modalitätsformen zählt man auch die Modalpartikeln, wie zum Beispiel „aber“ „denn“, „doch“, „eigentlich“, „einfach“, ,ja“, „nur“ „schon“ oder „,wohl“ (Gagel, 2015, 133-135; Weiss, 2016, 21).

Die dritte Lesart stellt die sprechaktbezogene Ebene dar, die in den folgenden Illokutionstypen wie Direktivum, Optativum, Interrogativum, Kommissivum, Expressivum und Deklarativum beschrieben werden kann (Weiss, 2016, 22).

Mit dem Direktivum werden vor allem Befehle und Aufforderungen ausgedrückt. Es kann auch durch die Verben wie „auffordern“, „befehlen“, „bitten“ oder „fragen" beschrieben werden.

Das Optativum bezeichnet Wunschsätze.

Das Interrogativum drückt Fragesätze aus, die entweder Entscheidungs- oder Ergänzungsfragen sein können.

Das Kommissivum bezeichnet eine zukünftige Handlung des Sprechers und kann mit den Verben wie „anbieten“, „schwören“, „vereinbaren“ oder „versprechen" beschrieben werden. Das Expressivum drückt einen psychischen Zustand des Sprechers aus, und kann durch die Verben wie „sich entschuldigen”, „danken”, „wünschen” oder „gratulieren” bezeichnet werden. Der letzte Illokuti- 
onstyp ist das Deklarativum, das sofortige Veränderungen am Zustand der Dinge zum Ausdruck bringt und mit Hilfe der Verben wie „entlassen“, „verurteilen“ oder „ernennen“ beschrieben werden kann (Gagel, 2015, 26-27).

\section{Untersuchungsmethodik}

In diesem Teil des Beitrags wird der Forschungsplan einer beginnenden Untersuchung dargestellt, die sich mit dem Leseverstehen in der Fremdsprache bzw. mit dem Textverstehen im Fremdsprachenunterricht in Bezug auf pragmalinguistische Indikatoren befasst. Außerdem wird in diesem Teil auch die Bedeutung der Pragmalinguistik im Kontext des Textverstehens vorgestellt. Diese Untersuchung wird mit den Schülern des Sekundarbereichs realisiert, die die deutsche Sprache als zweite Fremdsprache auf dem Niveau A2 nach dem GER für Sprachen lernen.

In erster Linie werden das Untersuchungsumfeld und das Untersuchungsziel der gegebenen Problematik geschildert und erklärt, danach die Untersuchungsmethoden beschrieben, aufgrund derer die Forschung während der nächsten zwei Jahren realisiert wird.

Das letzte Subkapitel präsentiert eine kurze Inhaltsanalyse von zwei Texten aufgrund der pragmalinguistischen Typologie. Die ausgewählten Texte sind vor allem Gebrauchstexte, die (von Hockicková-Stranovská-Weiss-Stančeková-FiczereSzabó, 2019) im Internet gefunden und modifiziert wurden. In den Mustertexten werden die einzelnen kognitiven Verknüpfungsebenen analysiert, die als wichtige Hilfsmittel beim Leseverstehen dienen.

\section{Text 1}

BRAVO erscheint jede Woche im Münchner Heinrich Bauer Verlag. BRAVO ist voll mit kurzen Texten und bunten Bildern, Kino-, Fernseh- und Modetipps, Infos über Film- und Popstars. Warum ist BRAVO die erfolgreichste unter den Jugendzeitschriften? Vielleicht ist sie einfach näher dran an den Teens, an ihren Wünschen, Unsicherheiten und Ängsten. „Bin ich zu dick?“, „Bin ich zu klein?“, ,WWie bekomme ich endlich eine Freundin?" - in der Pubertät kann die Liste solcher Fragen ziemlich lang werden. Viele Jugendliche wollen damit nicht zu ihren Eltern gehen.

\section{Text 2}

Liebe Pia, leider kann ich dich nicht abholen, deshalb musst du den Weg allein finden. Aber keine Angst, es ist ganz einfach! Du gehst vom Bahnhof geradeaus bis zum Fluss. Nicht über die Brücke gehen! Geh rechts den Fluss entlang und dann durch den kleinen Park bis zur Kirche. Da gehst du links in die Ringstraße. Gegenüber der Bäckerei ist die Hausnummer 53 und da wohne ich. 


\subsection{Untersuchungsumfeld und Untersuchungsziel}

Heutzutage gibt es immer mehr Menschen, nicht nur Studenten, sondern auch Erwachsenen, die Probleme mit dem Leseverstehen sowohl in der Muttersprache als auch in der Fremdsprache haben. Die vorliegende Forschung untersucht verschiedene Möglichkeiten zur Optimierung und Weiterentwicklung der Lesefertigkeit von Studenten im deutschen Sprachunterricht und gibt den Fremdsprachenlehrern Empfehlungen. Während der Untersuchung der Lesekompetenz geht man von der Theorie der Pragmalinguistik aus, die die kognitive Ebene detailliert analysiert und die sprachlichen Mittel beschreibt, die den Studierenden helfen können, den geschriebenen Text besser zu verstehen. Es ist sehr wichtig, sich mit der Pragmalinguistik zu beschäftigen, weil sie den Kommunikationsprozess der Individuen sowie ihre Wahrnehmung einer Fremdsprache beeinflusst.

Das Ziel dieser Untersuchung ist festzustellen, ob das Verstehen der fremdsprachigen Texte mit der kognitiven und soziolinguistischen Ebene zusammenhängt, die sich mit den Wahrnehmungs- und Denkprozessen befasst, sowie den sozialen Bedingungen der Sprachkommunikation, das Verhältnis der Sprache und Gesellschaft und das Verhältnis der Individuen zur Sprache untersucht. Außerdem soll bestimmt werden, welche kognitive Ebene für den Studenten im Text am deutlichsten ist. Die kognitive Ebene untersucht man mit unterschiedlichen Verknüpfungsebenen, die durch verschiedene Indikatoren beschrieben werden können.

\subsection{Untersuchungsmethode}

Während der Analyse verwendet man die qualitative Inhaltsanalyse des Textes, mit deren Hilfe die einzelnen kognitiven Verknüpfungsebenen in den Texten analysiert werden. Daneben verwendet man auch den Leistungstest, der eine quantitative Forschungsmethode ist, mit der man den Grad des Textverstehens feststellt. Es sollen vor allem Sachtexte und Gebrauchstexte des Alltags berücksichtigt werden, in denen man sich dem intensiven Lesen widmen wird und auch literarische Texte, in denen man sich auf das extensive Lesen konzentrieren wird. Aufgrund dieser Analyse werden Texte für das Interventionsprogramm vorgeschlagen, mit deren Hilfe die kommunikative Kompetenz und die Lesefertigkeit der Studenten im Fremdsprachenunterricht entwickelt werden können.

\subsection{Qualitative Inhaltsanalyse des Textes aufgrund der pragmalinguis- tischen Typologie}

In dem vorliegenden Teil werden die modifizierten Texte aufgrund der pragmalinguistischen Typologie von Sweetser (1990) analysiert, die in dem Teil 2 (Pragmalinguistik im Kontext des Textverstehens) beschrieben wurde. Beide Texte werden getrennt voneinander behandelt, wobei jede Verknüpfungsebene durch unterschiedliche Indikatoren charakterisiert wird. Im ersten Teil der Inhaltsanalyse wird der Text 1 detailliert untersucht. 


\section{propositionale Verknüpfungsebene}

BRAVO erscheint jede Woche im Münchner Heinrich Bauer Verlag.

BRAVO ist voll mit kurzen Texten und bunten Bildern, Kino-, Fernseh- und Modetipps, Infos über Film- und Popstars.

Die erwähnten Beispielsätze sind für die propositionale Verknüpfungsebene charakteristisch. Sie sind in der Indikativform ausgedrückt und stellen Beschreibungen nach Gagel (2015) und Weiss (2016) dar. In diesen Sätzen geht es darum, wo das Magazin BRAVO herausgegeben wird und was es beinhaltet.

\section{epistemische Verknüpfungsebene}

Vielleicht ist sie einfach näher dran an den Teens, an ihren Wünschen, Unsicherheiten und Ängsten.

Der folgende Beleg ist im Rahmen der epistemischen Ebene für das Estimativum typisch, das die Vermutung durch das Adverb „vielleicht“ ausdrückt (Gagel 2015, 29).

In der Pubertät kann die Liste solcher Fragen ziemlich lang werden. Viele Jugendliche wollen damit nicht zu ihren Eltern gehen.

Die nächsten zwei Sätze beschreiben die epistemische Verknüpfungsebene, weil sie Modalverben beinhalten, mit deren Hilfe in dem ersten Beispielsatz subjektive und in dem zweiten objektive Modalität zum Ausdruck gebracht wurde (Gagel 2015, 133; Weiss 2016, 21).

Vielleicht ist sie einfach näher dran an den Teens, an ihren Wünschen, Unsicherheiten und Ängsten.

Dieses Beispiel stellt auch die epistemische Ebene dar, weil eine Modalpartikel in dem Satz vorkommt, mit deren Hilfe mehr Informationen über die vorliegende Situation erlangt und Vermutung bzw. reale Möglichkeit ausgedrückt werden können. In dem gegebenen Beispielsatz betont die Modalpartikel „einfach“ die positive Seite des Magazins, was es den Teenagern anbietet, womit sie sich vertraut machen können (Gagel 2015, 134).

\section{sprechaktbezogene Verknüpfungsebene}

\section{„Bin ich zu dick?"}

„Bin ich zu klein?"

„Wie bekomme ich endlich eine Freundin?"

Die folgenden Fragesätze sind im Rahmen der sprechaktbezogenen Ebene für das Interrogativum charakteristisch, aus denen der dritte Satz Ergänzungs- und der erste und der zweite Satz Entscheidungsfragen sind, wie es Gagel (2015) in seinem Beitrag beschrieben hat. 
Warum ist BRAVO die erfolgreichste unter den Jugendzeitschriften?

Wenn man den ganzen Text liest, lässt sich feststellen, dass sich bei dem erwähnten Beleg nicht um das Interrogativum, sondern um den assertiven bzw. repräsentativen Sprechakt handelt. Der assertive Sprechakt nach Searle (1971) bedeutet, dass der Sprecher einen Sachverhalt darstellen und seinen Gesprächspartner über diesen Sachverhalt informieren möchte. Es geht hier sowohl um Vermutungen als auch um Feststellungen (Franz, 2014, 68). Aus dem Text kann man den Schluss ziehen, dass BRAVO die erfolgreichste aller Jugendzeitschriften ist.

In dem zweiten Teil der qualitativen Inhaltsanalyse wird der Text 2 aufgrund der pragmalinguistischen Typologie untersucht.

\section{propositionale Verknüpfungsebene}

Gegenüber der Bäckerei ist die Hausnummer 53 und da wohne ich.

Dieser Beleg steht für die propositionale Verknüpfungsebene, der in der Indikativform ausgedrückt ist. Der Satz schildert eine Beschreibung, wie es Gagel (2015) erläutert hat. Der Sprecher erklärt bzw. beschreibt, wo sich sein Haus genau befindet.

\section{epistemische Verknüpfungsebene}

Leider kann ich dich nicht abholen, deshalb musst du den Weg allein finden.

Das nächste Beispiel stellt das Identifikativum dar, das als ein Subtyp der epistemischen Ebene dient. Nach Gagel (2015) drückt das Adverb "leider" in diesem Beispielsatz das Gefühl des Sprechers aus, der seinem Gesprächspartner erklärt, dass er aus irgendeinem Grund inn nicht abholen kann.

Leider kann ich dich nicht abholen, deshalb musst du den Weg allein finden.

Der folgende Satz enthält Modalverben, die objektive Modalität von der Seite des Sprechers beschreiben (Gagel 2015, 133; Weiss 2016, 21). Der Sprecher ist nicht fähig, seinen Gesprächspartner abzuholen, deshalb ist dieser gezwungen, den Weg allein zu finden.

\section{sprechaktbezogene Verknüpfungsebene}

Aber keine Angst, es ist ganz einfach!

Du gehst vom Bahnhof geradeaus bis zum Fluss.

Nicht über die Brücke gehen!

Geh rechts den Fluss entlang und dann durch den kleinen Park bis zur Kirche. Da gehst du links in die Ringstraße.

Die letzten fünf Beispiele sind im Rahmen der sprechaktbezogenen Verknüpfungsebene für das Direktivum typisch. Mit Hilfe dieses Illokutionstyps werden in den 
erwähnten Sätzen Aufforderungen realisiert, mit Hilfe derer der Gesprächspartner dazu bewegt werden soll, in eine bestimmte Richtung zu gehen. Für das Direktivum ist noch ganz charakteristisch, dass das finite Verb im Imperativ steht, wie es in dem vierten Satz der Fall ist (Gagel 2015, 26-27).

\section{Zusammenfassung und Diskussion}

Aus der Analyse geht hervor, dass die ausgewählten Texte für das Verstehen und die Untersuchung auf der Grundlage der pragmalinguistischen Typologie geeignet sind, weil sie nach dem GER dem Niveau A2 entsprechende, semantisch und syntaktisch sinnvolle Sätze beinhalten. Mit Hilfe der pragmalinguistischen Analyse kann man die einzelnen kognitiven Verknüpfungsebenen bestimmen, für die verschiedene Indikatoren charakteristisch sind. Außerdem sind einige Sätze in unterschiedlichen Modi ausgedrückt, die das Textverstehen auch erleichtern können. Aber dazu braucht man schon ein gewisses Maß an grammatischem Wissen.

Die einzelnen Verknüpfungsebenen haben einen unterschiedlichen Schwierigkeitsgrad. Aufgrund der Belege kann man sehen, dass die propositionale Ebene neutral ist. Das bedeutet, dass die Sätze keine besonderen Indikatoren enthalten. Darüber hinaus lässt sich feststellen, dass diese Lesart ganz klar und verständlich für die Schüler sein kann. Dagegen fordert die Wahrnehmung sowohl der epistemischen als auch der sprechaktbezogenen Ebene schon eine grammatische und syntaktische Übersicht von den Studierenden. Sie sollten die Modalverben, die Modalpartikeln und auch die Adverbien, die bei der epistemischen Lesart vorkommen, kennen und beherrschen. Daneben ist es auch wichtig, die Bedeutung von einzelnen Verben zu verstehen, die eine entscheidende Rolle bei der Wahrnehmung der sprechaktbezogenen Ebene spielen.

In den ausgewählten Texten war es nicht möglich, alle Illokutionstypen (Evaluativum, Optativum, Kommissivum, Expressivum, Deklarativum) zu finden und zu analysieren, trotzdem gab es genügend Beispielsätze in den Mustertexten, mit deren Hilfe man eine gründliche qualitative Inhaltsanalyse der Texte durchführen konnte.

Aufgrund der Forschungsergebnisse lässt sich feststellen, dass sich die Lehrenden bei den Leseverstehensübungen vor allem darauf konzentrieren sollten, dem ausgewählten Niveau entsprechende Ausdrücke zu verwenden, die für das Textverstehen wesentlich sind, weil sie den Studierenden helfen genau das zu verstehen, was der Autor mitteilen und betonen möchte. Daneben darf man sich während der Kommunikation nicht nur auf die linguistische Kompetenz begrenzen. Es ist auch wichtig, die kommunikative Kompetenz auszubilden.

Während der weiteren Untersuchungen von Texten auf der Grundlage der pragmalinguistischen Typologie werden die bisherigen Forschungen mit den Forschungsergebnissen an Schulen miteinander verglichen, ob sich die Analysen in der realen Umgebung bestätigen. In den nächsten zwei Jahren werden wir uns noch intensiver in der Theorie der pragmalinguistischen Typologie vertiefen und auch unterschiedliche deutsche Texte auf dem Niveau A2 für die Lernenden des Sekundarbereichs vorbereiten, anhand derer ihre Lesefertigkeit nach den einzel- 
nen kognitiven Verknüpfungsebenen untersucht werden kann. Schließlich werden aufgrund dieser Untersuchung Texte für das Interventionsprogramm vorgeschlagen, mit deren Hilfe die kommunikative Kompetenz und die Lesefertigkeit der Lernenden im Fremdsprachenunterricht entwickelt werden können.

\section{Acknowledgement}

Diese Arbeit wurde von der Agentur für Forschung und dem Vertrag APV-17-0071 und VEGA 1/0062/19 unterstützt.

\section{Literaturverzeichnis}

Clarke, Paula - Truelove, Emma - Hulme, Charles - Snowling, Margaret Jean (2014): Developing Reading Comprehension. Hoboken, $\mathrm{NJ}$ : Wiley Blackwell.

Ehlers, Svantje (2006): Entwicklung von Lesekompetenz in der Fremdsprache. Berlin: De Gruyter. 31-38. p. [online].

http://babylonia.ch/fileadmin/user_upload/documents/2006-3-4/Baby3-4_06Ehlers. pdf Abgerufen am 22.11.2019

Ferraresi, Gisella (2011): Konnektoren im Deutschen und im Sprachvergleich: Beschreibung und grammatische Analyse. Tübingen: Narr. 127-155. p.

Franz, Norman (2014): Die Sprechakttheorie nach Austin und Searle: Äußerungen als Handlung. Hamburg: Diplomica Verlag.

Gadušová, Zdenka (2004): Teória a prax osvojovania cudzích jazykov: prijemca: dietáa. Nitra UKF.

Gagel, Sebastian (2015): Frühneuhochdeutsche Konnektoren - Entwicklungslinien kausaler Verknüpfungen auf dem Gebiet der Modalität. Diss. masch. Universität Erlangen - Nürnberg.

Gavora, Peter (2003): Modely a úrovne gramotnosti. In Gramotnost'. Vývin a možnosti jej didaktického usmerňovania. Bratislava: Univerzita Komenského. 171-181. p.

Gavora, Peter et al. (2008): Ako rozvijat' porozumenie textu u žiaka. Príručka pre učitela. Nitra: ENIGMA.

Günther, Britta - Günther, Herbert (2007): Erstsprache, Zweitsprache, Fremdsprache. Eine Einführung. 2. Auflage, Weinheim und Basel: Beltz Verlag.

Hirschnerová, Zuzana (2012): Slovenský jazyk pre 4. Ročník základných škôl metodická príručka. Bratislava: Aitec.

Hufeisen, Britta - Neuner, Gerhard (1999): Angewandte Linguistik für den fremdsprachlichen Deutschunterricht. Fernstudieneinheit 16. Kassel-München-Tübingen: Universität Kassel.

Kovárová, Dana - Kurtulíková, Alena (2010): Mimočítankové čítanie. Metodické poznámky. Bratislava: Orbis Pictus Istropolitana, spol. s.r.o.

Liptáková, Ĺudmila - Cibáková, Dana (2013): Edukačný model rozvíjania porozumenia textu v primárnom vzdelávaní. Prešovská univerzita v Prešove: Pedagogická fakulta. 
Searle, John Rogers (1971): Sprechakte. Ein sprachphilosophischer Essay. Frankfurt am Main: Suhrkamp Verlag.

Stranovská, Eva (2011): Psycholingvistika: determinanty osvojovania si a učenia sa cudzieho jazyka a kultúry. 1. vydanie. Brno: MSD.

Sweetser, Eve (1990): From etymology to pragmatics. Metaphorical and cultural aspects of semantic structure. Cambridge: Cambridge University Press.

Terpitková, Marianna (2013): Rozvoj čitatel'skej gramotnosti na hodinách anglického jazyka. Bratislava: Metodicko-pedagogické centrum.

Weiss, Ervín (2016): Das Stadtbuch von Schwedler - Möglichkeiten der Konnektorenforschung. Nitra: Filozofická fakulta. Univerzita Konštantína Filozofa v Nitre. 16-27. p. [online].

http://www.sung.sk/fotky10204/SZfG/2016_1/16_SZfG_1_2016.pdf Abgerufen am 20.11.2019

\section{Internetseite}

http://www.culture.gov.sk/extdoc/7090/bod_2_Koncepcia_rozvoja_kulturnych_a_citatelskych_kompetencii_deti, Abgerufen am 21.11. 2019 